\title{
An Extension of Heaviside's Operational Method of Solving Differential Equations.
}

\author{
By Dr Bevan B. Baker.
}

(Read 2nd May 1924. Received 1uth September 1924.)

\section{\$1. Introductory.}

An elegant symbolic method of solving differential equations was developed by Heaviside in his "Electrical Papers" and "Electromagnetic Theory," chiefly in connexion with problems concerning electric currents in net-works of wires. Attention has recently been called to the method by Bromwich, ${ }^{*}$ who applied it to a wider range of problems and gave an extension of Heaviside's formula; another generalisation of the formula has been abtained by Carson. $\dagger$

In the present paper a formula is obtained which contains the formulae of Heaviside, Bromwich and Carson as particular cases, and whose form is such that it may be readily applied to physical problems.

\section{$\$ 2 . \quad$ Deduction of the Formula.}

Consider a physical system which is governed by one or more differential equations; let $u$ be one of the dependent variables and $t$ one of the independent variables. Let $p$ be written for the operator $\partial / \partial t, p^{2}$ for $\partial^{2} / \partial t^{2}$ and so on and let $p$ be treated as a symbol obeying the ordinary laws of Algebra.

Suppose that after making this substitution a solution of the differential equation or equations can be obtained, satisfying any given boundary conditions, in the symbolic form

$$
u=\frac{F^{\prime}(p)}{\Delta(p)} P
$$

where $F(p)$ and $\Delta(p)$ denote polynomials in $p$, which are such that $\Delta(p)$ is of higher degree than $F(p)$, and $P$ is a function of the

- Phil Mag, 6th Ser., 37, (1919), p. 407.

+ Physical Review, 2nd Ser., 10, (1917), p. 217. 
independent variables. The method consists in interpreting this symbolic solution by obtaining an equivalent expression which no longer involves the operator $p$.

If $\Delta(p)$ be of degree $n$, and if its zeros be $\alpha_{1}, \alpha_{2}, \ldots, \alpha_{n}$, which we suppose all different, it is a well-known algebraic result that

$$
\frac{F(p)}{\Delta(p)}=\sum_{r=1}^{n} \frac{A_{r}}{p-\alpha_{r}}, \text { where } A_{r}=\frac{F^{\prime}\left(\alpha_{r}\right)}{\Delta^{\prime}\left(\alpha_{r}\right)}
$$

Let us suppose in the first place that $P=K t^{k} e^{\lambda t}$ where $K, k$ and $\lambda$ are independent of $t$. Let $z$ be the function obtained by operating on $P$ with the operator $\left(p-\alpha_{r}\right)^{-1}$, so that $z$ is a solution of the differential equation

$$
\left(\frac{\partial}{\partial t}-\alpha_{r}\right) z=K t^{k} e^{\lambda t}
$$

integrating this as an ordinary differential equation for $z$, we obtain

$$
\begin{aligned}
& z e^{-a_{r} t}=C+K \int t^{k} e^{\left(\lambda-a_{r}\right) t} d t \\
& =C+K e^{\left(\lambda-a_{r}\right) t}\left[\frac{t^{k}}{\lambda-a_{r}}-\frac{k t^{k-1}}{\left(\lambda-\alpha_{r}\right)^{2}}+\frac{k(k-1) t^{k-2}}{\left(\lambda-\alpha_{r}\right)^{3}}-\ldots+\frac{(-1)^{k} k !}{\left(\lambda-\alpha_{r}\right)^{k+1}}\right]
\end{aligned}
$$

where $C$ is an arbitrary constant of integration. We now take $z$ to be that solution of (3) which reduces to zero when $t=0$, so that

$$
\begin{array}{r}
z=K e^{\lambda t}\left[\frac{t^{k}}{\lambda-\alpha_{r}}-\frac{k t^{k-1}}{\left(\lambda-\alpha_{r}\right)^{2}}+\frac{k(k-1) t^{k-2}}{\left(\lambda-\alpha_{r}\right)^{3}}-\ldots+\frac{(-1)^{k} k !}{\left(\lambda-\alpha_{r}\right)^{k+1}}\right] \\
-\frac{(-1)^{k} k ! K e^{a_{r} t}}{\left(\lambda-\alpha_{r}\right)^{k+1}}, \ldots \ldots
\end{array}
$$

To obtain the result of operating on $P$ with $F(p) / \Delta(p)$ we multiply the expression (4) by $A_{r}$ and sum for all values of $r$ from 1 to $n$, giving for the value of $u$ which reduces to zero when $t=0$ the formula

$$
\begin{aligned}
u=K[ & e^{\lambda t}\left\{t^{k} \sum_{r=1}^{n} \frac{A_{r}}{\lambda-\alpha_{r}}-k t^{k-1} \sum_{r=1}^{n} \frac{A_{r}}{\left(\lambda-\alpha_{r}\right)^{2}}+k(k-1) t^{k-2} \sum_{r=1}^{n} \frac{A_{r}}{\left(\lambda-\alpha_{r}\right)^{3}}\right. \\
& \left.\left.-\ldots+(-1)^{k} k ! \sum_{r=1}^{n} \frac{A_{r}}{\left(\lambda-\alpha_{r}\right)^{k+1}}\right\}-(-1)^{k} k ! \sum_{r=1}^{n} \frac{A_{r} e^{\alpha_{r} t}}{\left(\lambda-\alpha_{r}\right)^{k+1}}\right] \ldots(5)
\end{aligned}
$$


Now suppose that $F(p) / \Delta(p)$ can be expanded in a series of positive powers of $(p-\lambda)$ in the form

$$
\frac{F(p)}{\Delta(p)}=N_{0}+N_{1}(p-\lambda)+N_{2}(p-\lambda)^{2}+N_{3}(p-\lambda)^{3}+\ldots \ldots
$$

From equation (2) we obtain also

$$
\begin{aligned}
\frac{F(p)}{\Delta(p)}=\sum_{r=1}^{n} \frac{A_{r}}{\lambda-\alpha_{r}}\left(1+\frac{p-\lambda}{\lambda-\alpha_{r}}\right)^{-1}=\sum_{r=1}^{n} & \frac{A_{r}}{\lambda-\alpha_{r}}-(p-\lambda) \sum_{r=1}^{n} \frac{A_{r}}{\left.\lambda-\alpha_{r}\right)^{2}} \\
& +(p-\lambda)^{2} \sum_{r=1}^{n} \frac{A_{r}}{\left(\lambda-\alpha_{r}\right)^{3}}-\ldots . .
\end{aligned}
$$

Comparing the coefficients of powers of $(p-\lambda)$ in these expansions we have

$$
\begin{aligned}
& N_{0}=\sum_{r=1}^{n} \frac{A_{r}}{\lambda-\alpha_{r}}, N_{1}=-\sum_{r=1}^{n} \frac{A_{r}}{\left(\lambda-\alpha_{r}\right)^{2}}, \ldots, \\
& N_{\imath}=(-1)^{s} \sum_{r=1}^{n} \frac{A_{r}}{\left(\lambda-\alpha_{r}\right)^{n+1}}, \ldots,
\end{aligned}
$$

and the solution (5) can be written in the form

$$
\begin{aligned}
& u=K\left[e ^ { \lambda t } \left\{N_{0} t^{k}+N_{1} k t^{k-1}+N_{2} k(k-1) t^{k-8}\right.\right.\left.+\ldots+N_{k} k !\right\} . \\
&\left.-(-1)^{k} k ! \sum_{r=1}^{n} \frac{A_{r}}{\left(\lambda-\alpha_{r}\right)^{k+1}} e^{a_{r} t}\right] \\
&=K\left[e^{\lambda t}\left\{N_{0} t^{k}+N_{1} \frac{d}{d t} t^{k}+N_{2} \frac{a^{2}}{d t=} t^{k}+\ldots+N_{k} \frac{d^{k}}{d t^{k}} t^{k}\right\}\right. \\
&\left.-(-1)^{k} k ! \sum_{r=1}^{n} \frac{F\left(\alpha_{r}\right) e^{a_{r} t}}{\left(\lambda-\alpha_{r}\right)^{k+1} \Delta^{\prime}\left(\alpha_{r}\right)}\right]
\end{aligned}
$$

It is then easy to extend the lesult to the case in which $P$ bas the form $f(t) e^{\lambda t}$ where $f(t)$ is a polynomial in $t$ of degree $m$, and we obtain the following general theorem :-

If $u$ be one of the dependent variables in a differential equation or system of differential equations and $t$ be one of the independent variables, and if by writing $\partial / \partial t=p$ and treating $p$ as an algebraic quantity a solution satisfying the boundary conditions can be obtained in the symbolic form

$$
u=\frac{F(p)}{\Delta(p)} P
$$


where $F(p)$ and $\Delta(p)$ are polynomials in $p$ such that $\Delta(p)$ is of higher degree than $F(p)$, and $P$ is a function of $t$ (and of the other independent variables) of the form

$$
P=f(t) e^{\lambda t}, \text { where } f(t)=C_{0}+C_{1} t+C_{2} t^{2}+\ldots+C_{k} t^{k} ;
$$

then the solution which satisfies the prescribed boundary conditions and which reduces to zero when $t=0$ is given by

$$
\begin{array}{r}
u=e^{\lambda t}\left[N_{0} f(t)+N_{1} f^{\prime \prime}(t)+N_{\mathrm{g}} f^{\prime \prime}(t)+\ldots+N_{k} f^{k}(t)\right] \\
+\sum_{r=1}^{n} \frac{F\left(\alpha_{r}\right)}{\Delta^{\prime}\left(\alpha_{r}\right)} e^{a_{r} t}\left[\frac{C_{0}}{\alpha_{r}-\lambda}+\frac{C_{1}}{\left(\alpha_{r}-\lambda\right)^{2}}+\frac{C_{3} \cdot 2 !}{\left(\alpha_{r}-\lambda\right)^{3}}+\frac{C_{3} \cdot 3 !}{\left(\alpha_{r}-\lambda\right)^{4}}\right. \\
\left.+\ldots+\frac{C_{k} \cdot k !}{\left(\alpha_{r}-\lambda\right)^{k+1}}\right],
\end{array}
$$

where $N_{0}, N_{1}, \ldots$ are the coefficients in the expansion of $F(p) / \Delta(p)$ in a series of powers of $(p-\lambda)$ in the form

$$
\frac{F(p)}{\Delta(p)}=N_{0}+N_{1}(p-\lambda)+N_{2}(p-\lambda)^{2}+\ldots
$$

and $\alpha_{1}, \alpha_{2}, \ldots, \alpha_{n}$ are the roots of $\Delta(p)=0$.

The formula (6) may be written in the alternative form

$$
\begin{aligned}
u & =e^{\lambda t}\left[N_{0}+N_{1} \frac{d}{d t}+N_{2} \frac{d^{2}}{d t}+\ldots+N_{k} \frac{d^{k}}{d t^{k}}\right] f(t) \\
& +\sum_{r=1}^{n} \frac{F\left(\alpha_{r}\right)}{\Delta^{\prime}\left(\alpha_{r}\right)} e^{\alpha_{r} \ell}\left[f\left(\frac{d}{d s}\right)\left(\frac{1}{\alpha_{r}-s}\right)\right]_{s=\lambda}
\end{aligned}
$$

or

$$
u=e^{\lambda t}\left[\frac{F\left(\lambda+\frac{d}{d t}\right)}{\Delta\left(\lambda+\frac{d}{d t}\right)} f^{\prime}(t)\right]+\sum_{r=1}^{n} \frac{F\left(\alpha_{r}\right)}{\Delta^{\prime}\left(\alpha_{r}\right)} e^{a_{r} t}\left[f\left(\frac{d}{d s}\right)\left(\frac{1}{a_{r}-8}\right)\right]_{\delta=\lambda}
$$

where the expression $F^{\prime}\left(\lambda+\frac{d}{d t}\right) / \Delta\left(\lambda+\frac{d}{d t}\right)$ is supposed to be expanded in a series of powers of $\frac{d}{d t}$.

It is easy to modify the result for the case in which the roots of $\Delta(p)=0$ are repeated. 
Although the theorem has only been proved when $F(p)$ and $\Delta(p)$ are polynomials in $p$, it is readily seen that the formula may also be used when more general types of functions take the place of these polynomials, though a formal proof in such cases is more troublesome; such cases will be illustrated in the examples which follow.

Evidently the result will also apply when the function $f(t)$ is in the form of an infinile series of powers of $t$, provided that the series on the right hand side of (6) are convergent.

When $P$ is a constant, say $C$, the formula reduces to Heaviside's result,

$$
u=C\left[\frac{F(0)}{\Delta(0)}+\sum_{r=1}^{n} \frac{F\left(\alpha_{r}\right)}{\Delta^{\prime}\left(\alpha_{r}\right)} \frac{\alpha_{r^{t}}}{\alpha_{r}}\right] .
$$

When $P=C e^{\lambda t}$, the formula becomes

$$
u=C\left[\frac{F(\lambda)}{\Delta(\lambda)} e^{\lambda t}+\sum_{r=1}^{n} \frac{F\left(\alpha_{r}\right)}{\Delta^{\prime}\left(\alpha_{r}\right)} \frac{e^{\alpha_{r}}}{\left(\alpha_{r}-\lambda\right)}\right]
$$

which is the result given by Carson (loc. cit.)

When $P=C t$, we obtain the formula

$$
u=C\left[N_{0} t+N_{1}+\sum_{r=1}^{n} \frac{F^{\prime}\left(\alpha_{r}\right)}{\Delta^{\prime}\left(\alpha_{r}\right)} \frac{e^{a_{r}}}{\alpha_{r}^{2}}\right]
$$

which is Bromwich's expression (loc. cit.).

\$3. Example 1. The interaction of Two Equal Coils.

As a first illustration of the method we will consider the effect of switching an electromotive force $E$ into one of two coils which, to simplify the algebra we will take to be equal in all respects. Let $R$ be the resistance, $L$ the self-inductance and $M$ the mutual inductance of the two coils and let the current in the primary coil be $x$, that in the secondary being $y$; the equations to be satisfied are therefore

$$
L \frac{d x}{d t}+M \frac{d y}{d t}+R x=E, M \frac{d x}{d t}+L \frac{d y}{d t}+R y=0 .
$$


Writing $p$ for $\frac{d}{d t}$ these become

$$
(L p+R) x+M p y=E, M p x+(L p+R) y=0
$$

and therefore

$$
x=\frac{(L p+R) E}{(L p+R)^{2}-M^{2} p^{2}}, y=-\frac{M p E}{(L p+R)^{2}-M^{2} p^{2}} .
$$

Let the electromotive force $E$ be switched into the primary circuit at the instant $t=0$, and let it be represented by

$$
E=f(t) e^{\lambda t} \text { where } f(t)=M_{0}+M_{1} t+M_{2} t^{2}+\ldots+M_{m} t^{m} .
$$

To obtain the current $x$ we have to interpret the effect of the operator $F(p) / \Delta(p)$ acting on the function $E$, where

$$
F(p)=L p+R \text { and } \Delta(p)=(L p+R)^{2}-M^{2} p^{2} .
$$

Writing $\xi=p-\lambda$, the operator $F(p) / \Delta(p)$ can be written in the form

$$
\begin{gathered}
\frac{A \xi+B}{C \xi^{2}+D \bar{D}+F}=\phi(\xi) \text { say, where } \\
A=L, B=L \lambda+R, C=L^{2}-M^{2}, D=2\left\{\left(L^{2}-M^{2}\right) \lambda+L R\right\}, \\
F=(L \lambda+R)^{2}-M^{2} \lambda^{2} .
\end{gathered}
$$

Expanding the function $\phi(\xi)$ in a series of powers of $\xi$ by Maclaurin's theorem we obtain

$$
\phi(\xi)=\frac{B}{F}+\frac{(A F-B D)}{F^{2}} \xi+\frac{\left(B D^{2}-C B F-A F D\right)}{F^{3}} \xi^{2}+\ldots
$$

the later coefficients in the series being determined from the recurrence formula

$$
F \phi^{n}(0)+n D \phi^{n-1}(0)+n(n-1) C \phi^{n-2}(0)=0 .
$$

In the notation of $\S 2$ we have therefore

$$
N_{0}=\frac{B}{F}, N_{1}=\frac{A F-B D}{F}, N_{2}=\frac{B D^{2}-C B F-A D F}{F}, \ldots
$$


therefore

Again the roots of $\Delta(p)=0$ are $\alpha=-\frac{R}{L+M}, \beta=-\frac{R}{L-M}$ and

$\frac{F(\alpha)}{\Delta^{\prime}(\alpha)}=\frac{L \alpha+R}{2 L(L \alpha+R)-2 M^{2} \alpha}=\frac{L \alpha+R}{\left.2 !\left(L^{2}-M^{2}\right) \alpha+L R\right\}}=\frac{1}{2(L+M)}$,

$\frac{F(\beta)}{\Delta^{\prime}(\beta)}=\frac{L \beta+R}{2\left\{\left(L^{2}-M^{2}\right) \beta+L R\right\}}=\frac{1}{2(L-M)}$.

Applying the formula of $\S 2$ the current in the primary circuit is given by

$$
\begin{gathered}
x=e^{\lambda t}\left[N_{0} f(t)+N_{1} \frac{d}{d t} f(t)+N_{2} \frac{d^{2}}{d t^{2}} f(t)+\ldots+N_{m} \frac{d^{m}}{d t^{m}} f(t)\right] \\
+\frac{e^{a t}}{2(L+M)}\left[\frac{M_{0}}{\alpha-\lambda}+\frac{M_{1}}{(\alpha-\lambda)^{2}}+\frac{M_{2} \cdot 2 !}{(\alpha-\lambda)^{3}}+\frac{M_{3} \cdot 3 !}{(\alpha-\lambda)^{4}}+\ldots+\frac{M_{m} \grave{m !}}{(\alpha-\lambda)^{m+1}}\right] \\
+\frac{e^{\beta t}}{2(L-M)}\left[\frac{M_{0}}{\beta-\lambda}+\frac{M_{1}}{(\beta-\lambda)^{2}}+\frac{M_{2} \cdot 2 !}{(\beta-\lambda)^{3}}+\frac{M_{3} \cdot 3 !}{(\beta-\lambda)^{4}}+\ldots+\frac{M_{m} m !}{(\beta-\bar{\lambda})^{m+1}}\right]
\end{gathered}
$$

To obtain the current in the secondary circuit we consider the operator $F(p) / \Delta(p)$ where $F(p)=-M p$ and $\Delta(p)=(L p+R)^{2}-M^{2} p^{2}$ and obtain in this case

$$
\begin{aligned}
N_{0}^{\prime} & =-\frac{M \lambda}{F}, N_{1}^{\prime}=-\frac{M(F-D \lambda)}{F^{2}}, N_{2}^{\prime}=-\frac{M\left(D^{2} \lambda-C F \lambda-D F\right)}{F^{3}}, \ldots, \text { and } \\
y & =e^{\lambda t}\left[N_{0}^{\prime} f(t)+N_{1}^{\prime} \frac{d}{d t} f(t)+N^{\prime} \frac{l^{2}}{t^{2}} f(t)+\ldots+N_{m}^{\prime} \frac{d^{m}}{d t^{m}} f(t)\right] \\
& +\frac{e^{\alpha t}}{2(L+M)}\left[\frac{M_{0}}{\alpha-\lambda}+\frac{M_{1}}{(\alpha-\lambda)^{2}}+\frac{M_{2} \cdot 2 !}{(\alpha-\lambda)^{a}}+\frac{M_{3} \cdot 3 !}{(\alpha-\lambda)^{4}}+\ldots+\frac{M_{m} \cdot m !}{(\alpha-\lambda)^{m+1}}\right] \\
& -\frac{e^{\beta t}}{2(L-M)}\left[\frac{M_{0}}{\beta-\lambda}+\frac{M_{1}}{(\beta-\lambda)^{2}}+\frac{M_{2} \cdot 2 !}{(\beta-\lambda)^{3}}+\frac{M_{3} \cdot 3 !}{(\beta-\lambda)^{4}}+\ldots+\frac{M_{m} \cdot m !}{(\beta-\lambda)^{m+1}}\right]
\end{aligned}
$$

By putting $\lambda=\iota \mu$ and then taking the real parts of these expressions for $x$ and $y$ we should obtain the currents in the two circuits when the electromotive force in the primary circuit is of the form

$$
E=f(t) \cos \mu t, \text { where } f(t)=M_{0}+M_{1} t+M_{2} t^{2}+\ldots+M_{m} t^{m},
$$


\$4. Example 2. The conduction of Heat throngh a Block of Finite Thickness.

Consider the conduction of heat in an infinite block of conducting material of thickness $l$, whose initial temperature is zero and whose faces are parallel and are maintained at temperatures $f_{1}(t)$ and $f_{2}(l)$ from the instant $t=0$ onwards.

The axis of $x$ being taken perpendicular to the bounding planes, the temperature $u$ satisfies the differential equation

$$
k \frac{\partial^{2} u}{\partial x^{2}}=\frac{\partial u}{\partial t}
$$

where $k=K / \rho \sigma, K$ being the thermal conductivity, $\rho$ the density and $\sigma$ the specific heat of the substance composing the block; subject to the boundary conditions:- $u=f_{1}(t)$ for $x=0$ and $u=f_{\varepsilon}(t)$ for $x=l$, for all positive values of $t$.

Writing $p$ for $\partial / \partial t$ and putting $p=k q^{2}$, the solution satisfying the assigned boundary conditions is obtained in the symbolic form

Now suppose

$$
u=\frac{\sinh q(l-x)}{\sinh q l} f_{1}(t)+\frac{\sinh q x}{\sinh q l} f_{2}(t) .
$$

$$
f_{1}(t)=\left(A_{0}+A_{1} t+A_{2} t^{2}\right) e^{\lambda_{1} t}, f_{2}(t)=\left(B_{0}+B_{1} t+B_{2} t^{8}\right) e^{\lambda_{2} t},
$$

and write $u=u_{1}+u_{2}$, where $u_{1}$ is the part of $u$ arising from $f_{1}(t)$ and $u_{2}$ is the part arising from $f_{2}(t)$.

Consider first $u_{2}$ and, in the notation of $\S 2$, take

$$
F(p)=\sinh q x / q, \Delta(p)=\sinh q l / q ;
$$

then expanding $F(p) / \Delta(p)$ in a series of powers of $\left(p-\lambda_{2}\right)$ we obtain

$$
F(p) / \Delta(p)=N_{0}+N_{1}\left(p-\lambda_{2}\right)+N_{\mathrm{g}}\left(p-\lambda_{2}\right)^{2}+\ldots,
$$

where

$$
N_{0}=\frac{\sinh \beta_{2} x}{\sinh \beta_{2} l}, N_{1}=\frac{x \cosh \beta_{2} x-l \operatorname{coth} \beta_{2} l \sinh \beta_{2} x}{2 \sqrt{k \lambda_{2} \sinh \beta_{2} l}}
$$

$$
N_{2}=\frac{\left(x^{2}-l^{2}\right) \sinh \beta_{q} l \sinh \beta_{2} x-\left(\beta_{2}^{-1} \sinh \beta_{2} l+2 l \cosh \beta_{2} l\right)\left(x \cosh \beta_{2} x-l \operatorname{coth} \beta_{2} l \sinh \beta_{2} x\right)}{8 k \lambda_{2} \sinh ^{2} \beta_{2} l}
$$

where $\beta_{2}=\sqrt{\lambda_{2} / k}$. 
The roots $\alpha_{0,} \alpha_{1}, \alpha_{2}, \ldots$ of $\Delta(p)=0$ occur when $q l=\iota r \pi$, where $r$ is zero or a positive integer, ${ }^{*}$ so that $\alpha_{r}=-k r^{2} \pi^{2} / l^{2}$; we obtain

$$
\frac{F\left(a_{r}\right)}{\Delta^{\prime}\left(a_{r}\right)}=-(-1)^{r} \frac{2 k r \pi}{l^{2}} \sin \frac{r \pi x}{l} \text {. }
$$

It follows from the formula of $\$ 2$, that

$$
\begin{gathered}
u_{2}=\left\{N_{0} B_{0}+N_{1} B_{1}+2 N_{2} B_{2}+\left(N_{0} B_{1}+2 N_{1} B_{2}\right) l+N_{0} B_{2} t^{2}\right\} e^{\lambda_{2} t} \\
+\sum_{r=0}^{\infty}(-1)^{r} \frac{2 k r \pi}{l^{2}} \sin \frac{r \pi x}{l} e^{\frac{-k r^{2} \pi^{2} t}{l^{2}}} \\
\left\{\frac{B_{0}}{\lambda_{2}+k r^{2} \pi^{2} / l^{2}}-\frac{B_{1}}{\left(\lambda_{2}+k r^{2} \pi^{2} / l^{2}\right)^{2}}+\frac{2 B_{2}}{\left(\lambda_{2}+k r^{2} \pi^{2} / l^{2}\right)^{3}}\right\} .
\end{gathered}
$$

The value of $u_{1}$ is obtained from this by changing $x$ into $l-x$ and replacing $B_{0}, B_{1}, B_{2}$ by $A_{0}, A_{1}, A_{2}$, respectively, and $\lambda_{2}$ by $\lambda_{1}$. By adding the value of $u_{1}$ so obtained to the above expression for $u_{a}$ we obtain the required temperature $u$.

By writing $\lambda_{1}=\iota \sigma_{1}$ and $\lambda_{2}=\iota \sigma_{2}$ and taking the real parts, the formulae give the temperature at any point when, from the instant $t=0$ onwards, the bounding planes are maintained at temperatures

$$
f_{1}(t)=\left(A_{0}+A_{1} t+A_{3} t^{2}\right) \cos \sigma_{1} t, f_{2}(t)=\left(B_{0}+B_{1} t+B_{2} t^{2}\right) \cos \sigma_{2} t .
$$

- The values for which $r$ is a negative integer provide no new roots of $\Delta(p)=0$. 